\title{
Potentia Dei y su concurso en el mundo natural según Juan Duns Escoto
}

\section{[Potentia Dei and Its Concursus in the Natural World According to John Duns Scotus]}

\author{
ENRIQUE SANTIAGO MAYOCCHI \\ Universidad Católica Argentina \\ Consejo Nacional de Investigaciones Científicas y Técnicas \\ emayocchi@gmail.com
}

\begin{abstract}
Resumen: Juan Duns Escoto configura su pensamiento filosófico desde una perspectiva cristiana, en la que la acción divina omnipotente crea y conserva las criaturas. Toda obra del creador ad extra es contingente, de manera que la realidad física recibe ese añadido en el nivel ontológico y operativo. Los objetivos de este trabajo son dos: determinar el alcance y la modalidad en que la potentia Dei actúa sobre el mundo natural según el Doctor Sutil y resolver una posible inconsistencia cuando este pensador analiza la capacidad del homo viator para alcanzar certeza en el conocimiento experiencial.

Palabras clave: omnipotencia, creación, causalidad, conocimiento, certeza
\end{abstract}

\begin{abstract}
John Duns Scotus molds his philosophical thought from a Christian perspective, in which an omnipotent divine action creates and preserves creatures. All what the creator produces ad extra is contingent, so whatever is added to physical reality belongs to an ontological and operational level. This paper aims to determine the extent and manner in which, according to the Doctor Subtilis, the potentia Dei operates on the natural world and to resolve a possible inconsistency when Scotus analyzes the ability of the homo viator to achieve certainty in the experiential knowledge.
\end{abstract}

Key words: omnipotence, creation, causality, knowledge, certainty

El desarrollo del pensamiento de Duns Escoto está marcado, en cierta medida, por las condenas que se formularon contra el aristotelismo en 1277 y con las que se defiende el poder divino, en particular sobre el mundo natural. Este trabajo tiene dos objetivos: determinar el alcance y la modalidad en que la potentia Dei actúa sobre el mundo natural según el Doctor Sutil y resolver una posible inconsistencia cuando este pensador analiza la capacidad del homo viator para alcanzar la certeza en el conocimiento experiencial. Para lograr estos fines divido mi exposición en tres secciones, en las que abordaré (1) el tema de la omnipotencia divina como causa de la creación a partir de una elección entre infinitos órdenes posibles y el modo contingente de tal acción y orden elegido; (2) la concurrencia específica entre la causa primera universal y los agentes físicos, así como las consecuencias que esto conlleva y, por úl-

Diánoia, volumen LXIII, número 80 (mayo de 2018): pp. 3-27. 
timo, (3) el problema de la certeza en el conocimiento experiencial del mundo físico en diálogo con una crítica contemporánea.

\section{Omnipotencia divina y creación}

El pensamiento cristiano que adopta Duns Escoto afirma que el mundo natural se creó ex nihilo, y este dato se sabe por medio de la revelación. No sin dificultades, los escolásticos desarrollan el análisis filosófico desde las categorías aristotélicas de acto y potencia para ofrecer una explicación racional al peculiar cambio que implica ese pasaje de no ser a ser. Con la idea aviceniana de que sólo puede ser hecho aquello que antes fue posible, ${ }^{1}$ el Doctor Sutil ve la necesidad de aclarar el origen de los posibles y su conexión con el acto creador. Este camino me llevará a explicar en primer término la conexión entre los posibles y la mente divina, y a continuación el modo en que esta última se relaciona con la voluntad como principio que determina la existencia de algunos posibles. Finalizaré la sección con una exposición sobre el fundamento de la contingencia ontológica y operacional de los seres creados.

Duns Escoto aborda el origen de los posibles a partir de dos dificultades que se tienen en cuenta para formular su propuesta personal. Por un lado, si el origen de los posibles presupone o no algo posible: la respuesta no puede ser afirmativa porque conduciría al infinito y la solución negativa lleva a suponer una creación ad intra en la mente divina, lo que no es posible por su condición de ser inmutable. Por otro lado, Escoto toma en cuenta la doctrina de Enrique de Gante, ${ }^{2}$ que se contrapone a la posición anterior y explica la creación como una recepción del esse existentiae dado por la causa eficiente primera al ser posible, cuyo esse essentiae se encontraba en potencia y era, antes de recibir la existencia, un verdadero esse; sin embargo, tampoco es una respuesta satisfactoria, puesto que en ese caso se destruiría el pasaje del no ser al ser a favor de la transición de un ser (esencial) a otro ser (existencial), con lo cual caeríamos en una reducción de la creación al mero cambio del tipo alteración y, en definitiva, ad esse secundum quid.

Para Escoto, la solución se encuentra en el hecho de que el creador "produce algo de la nada simpliciter y, sin embargo, presupone un ser

${ }^{1}$ Cfr. Avicena, Liber de philosophia prima sive scientia divina, tratado IV, capítulo 2, líneas 52-53 (Van Riet 1977, p. 208): "Todo lo que comienza a existir [esse], antes de que exista, es necesario que sea posible en sí" [en adelante, todas las traducciones son mías].

${ }^{2}$ Cfr. Enrique de Gante, Summae quaestionum ordinariarum, art. 21, quaes. 4, (Badius 1520, vol. I, fs. 126v-128v).

Diánoia, vol. LXIII, no. 80 (mayo de 2018). 
posible que tiene ser secundum quid". ${ }^{3}$ En efecto, el ser posible en la mente divina no se crea, sino que se "produce" como un ser secundum quid, que es el estatus ontológico propio de todo esse intelligibile. Para evitar la objeción de que ocurre una mutación ad intra, debemos tener en cuenta que el Doctor Sutil explica esa "producción" de los posibles según instantes de naturaleza en el interior de la esencia divina, es decir, según un orden de anterioridad y posterioridad que de ninguna manera es temporal ni termina en una realidad nueva y distinta de la causa. Podemos resumir esto de la siguiente manera: 1) Dios conoce su propia esencia; 2) conoce y produce in esse intelligible los infinitos modos posibles en que ella puede imitarse; 3) compara su esencia con los posibles, y 4) conoce reflexivamente la relación causada en el instante anterior. ${ }^{4}$ La idea de que los posibles aparecen en el segundo instante de naturaleza pretende señalar el orden lógico del intelecto divino. En efecto, los posibles son los infinitos modos en que la esencia divina se reconoce como imitable "luego" de conocerse a sí misma.

Los posibles no poseen un ser en sí, sino que "en la mente divina esas naturalezas tienen sólo un esse cognitum, un ser que no es absoluto, sino relativo, dado que implica necesariamente una relación con Dios como conocedor; y, entendido bajo esta relación con el conocimiento de Dios, es disminuido", 5 es decir, no poseen un ser real antes de la creación que realiza libremente la voluntad divina, sino que manifiestan la relación de un atributo con la esencia divina. Recordemos que la noción unívoca de ens que sostiene Escoto le permite afirmar que los posibles en la mente divina son entes cuando se interpreta el nombre "comunísimamente". En Quaestiones quodlibetales 3 se distinguen tres sentidos en los que se puede entender el término "ente": estrictamente (toda sustancia — ser real, absoluto y per se-), comúnmente (sustancia, cantidad y cualidad —ser real y absoluto-) y comunísimamente, "en cuanto se extiende a todo lo que no es 'nada'". 6 A continuación, Escoto explica que "nihil" puede entenderse como lo que incluye contradicción y por lo tanto no puede tener ser ni dentro ni fuera del entendimiento, esto es, es lo imposible. En constrante, ens puede decirse de "todo lo que no incluye contradicción" y aplicarse así al ente de razón y al ente real, o sólo a "todo lo que es o puede ser fuera del alma", es

${ }^{3}$ J. Duns Escoto, Lectura in sententiarum, II.1.2 $\S 81$ (Commissio Scotistica [en adelante, CS] XVIII, p. 26).

${ }^{4}$ Cfr. J. Duns Escoto, Ordinatio, I.35 § 32 (CS VI, p. 258); Reportatio parisiensis examinata, I-A.36.1.1-2 §§ 77-78 (Wolter Bychkov II, p. 406).

${ }^{5}$ Maurer 1950, pp. 221-222.

${ }^{6}$ J. Duns Escoto, Quaestiones quodlibetales, $3.1 \S 7$ (Alluntis 1968, p. 93). 
decir, al ente real. El primer sentido permite extender el uso de ens a todo aquello que está en el intelecto aunque no exista en la realidad, y en este sentido comunísimo es como los posibles en la mente divina se llaman ens deminutum. La idea de "ser disminuido" indica la tensión entre el ser posible-potencial y el ser real-actual y se aleja así de un esse essentiae real en el interior de la esencia divina antes de la creación. "Nada, pues, es creado que no haya tenido antes ser entendido y querido, y en el ser entendido fue posible formalmente [...] y entonces se encontraba como en potencia próxima para poder ser objeto de la omnipotencia y ser puesto en el ser simpliciter."7

Antes de pasar al análisis del acto creador por el que los posibles reciben existencia, es necesario abordar el modo particular de principiar que tienen para Escoto el intelecto y la voluntad de Dios. En Questiones super libros Metaphysicorum IX.15, concluye que todo principio activo se comprende según dos modalidades, "naturaleza" y "voluntad", con lo cual realiza una transformación de las potencias activas aristotélicas, ${ }^{8}$ racionales e irracionales, mediante el criterio del modo en que éstas "elicitan"9 sus operaciones.

Una potencia [activa] o está determinada por sí a obrar, de manera que por su parte no puede no obrar cuando no se ve impedida por algo extrínseco o no está determinada por sí, sino que puede obrar este acto o el acto opuesto, [y] también [puede] obrar o no obrar. La primera potencia se llama comúnmente "naturaleza", la segunda se denomina "voluntad". ${ }^{10}$

El principio "naturaleza" obra con necesidad y siempre de la misma manera cuando el objeto se hace presente, siempre y cuando no se vea impedido por algo extrínseco. Sus acciones nunca podrán ser opuestas porque están determinadas según cada quidditas, pero sí sus efectos, ya que una misma actividad puede producir distintos resultados según

${ }^{7}$ Ordinatio, II.1.2 § 83 (CS VII, pp. 43-44).

${ }^{8}$ Cfr. González Ayesta 2007, p. 16.

${ }^{9} \mathrm{El}$ verbo "elicere", que no tiene un equivalente inmediato en castellano, es un término técnico con el cual Escoto designa las actividades propias de las potencias activas, inteligencia y voluntad, por las que producen una actividad inmanente, intelección y volición, términos que el Doctor Sutil entiende como cualidades en el alma; es decir, "elicitar" es la producción de un término en el género de la cualidad (y no de la categoría acción) por el cual conocemos o queremos un objeto. Cfr. Quaestiones super libros Metaphysicorum Aristotelis, IX.3-4 § 49 (OPh IV, pp. 557558); Cross 2008, pp. 278-279.

${ }^{10}$ J. Duns Escoto, Quaestiones super libros Metaphysicorum Aristotelis, IX.15 § 22 (OPh IV, pp. 680-681).

Diánoia, vol. LXIII, no. 80 (mayo de 2018). 
el sujeto que la recibe; por ejemplo, el Sol puede resecar con su calor el barro y derretir el hielo. Todo intelecto "elicita" su operación de manera necesaria, pues está determinado para conocer y no puede dejar de hacerlo por sí mismo. De este modo, el intelecto divino conoce necesariamente $^{11}$ todo el ámbito extensible con el ser, es omnisciente, y este conocimiento "es una condición necesaria para la creación libre de todo ser contingente". ${ }^{12}$

Por otro lado, el modo de obrar propio del principio activo "voluntad" es la contingencia, ya que tiene la capacidad de autodeterminación y en esto radica su libertad. La voluntad en el homo viator puede ser libre respecto de actos, objetos y efectos opuestos. Sin embargo, Escoto niega que la voluntad divina sea libre para realizar actos opuestos en un sentido diacrónico, porque esto implica mutabilidad e imperfección. En efecto, por la eternidad y simplicidad que la caracterizan sólo tiene un acto volitivo que se identifica con ella misma y, para asegurar que su elección es libre, Escoto explica que en su eternidad mantiene la posibilidad de un acto opuesto en sentido sincrónico. Para explicarlo, propone como ejemplo la proposición "la voluntad divina queriendo en un instante eterno que la piedra exista [esse] puede no querer [nolle] en ese mismo instante que la piedra exista [esse]". ${ }^{13}$ A continuación, explica que esta afirmación no es contradictoria si se comprende in sensu divisionis, es decir, como la conjunción de dos proposiciones de las que sólo se aplica el operador modal a la segunda, con lo que resulta la proposición: "La voluntad divina quiere en un instante eterno que la piedra sea y la voluntad divina puede no querer en ese mismo instante que la piedra sea". De esta manera, obtenemos una proposición actual y otra posible, ambas verdaderas al mismo tiempo. Este nuevo modo de aplicar el sentido dividido fortalece la conexión entre querer, libertad y contingencia, en la medida en que "querer algo es un acto libre, porque no querer es posible al mismo tiempo. De tal manera que la libertad de la voluntad se vuelve equivalente a la contingencia sincrónica de las voliciones". ${ }^{14}$ De aquí se desprende la noción específica de contingencia que utiliza Escoto, la cual se caracteriza por pertenecer al ámbito de lo factual; lo que, siendo, puede dejar de ser o ser de otra manera.

Un análisis paralelo es el de la libertad en relación con objetos opuestos, "la voluntad divina por una única volición quiere eternamente que

${ }^{11}$ Cfr. Lectura, I.39.1-5 § 43 (CS XVII, p. 492): “Todo aquello que [el intelecto divino] conoce antes del acto de la voluntad, lo conoce necesaria y naturalmente".

12 Ross y Bates 2003, p. 214

${ }^{13}$ Lectura, I.39.1-5 § 54 (CS XVII, p. 497).

${ }^{14}$ Vos et al. 1994, p. 123. 
la piedra exista [esse] y puede eternamente querer que la piedra no exista [non esse]". ${ }^{15}$ También esto debe comprenderse in sensu divisionis y de manera sincrónica, como en el caso de los actos opuestos. En esta misma línea, Escoto no encuentra problemas para explicar el concurso de la voluntad divina en la creación porque, aunque puedan darse en el mundo objetos opuestos de manera diacrónica, son queridos por un mismo acto volitivo y sin implicar mutación alguna, ya que "Dios, por medio de su simple acto volitivo, puede querer objetos opuestos [y] ellos pueden encontrarse sucesivamente en la línea temporal, de tal manera que Dios simultáneamente quiere $b$ para el momento $1, \mathrm{y}-b$ para el momento 2 , y así sucesivamente". ${ }^{16}$

Si retomamos ahora el tema de la creación, afirmé que la omnipotencia divina tiene como objeto propio a los infinitos posibles que produce el intelecto divino como modos imitables de su esencia en cuanto que creables o, lo que es lo mismo, compatibles con la existencia y el esse simpliciter. En este sentido, para Escoto "crear no es pasar de un orden mental a un orden real, sino de la nada a la existencia". ${ }^{17} \mathrm{El}$ acto creador consiste en una elección libre de la voluntad divina por la que determina el ser real de algunos posibles. Esta determinación no está motivada desde afuera ni por una carencia de perfección, sino por la superabundante suficiencia ${ }^{18}$ de la misma voluntad que se autodetermina al amar de manera gratuita en un acto infinitamente libre. "En el hecho de que Dios puede querer, en el mismo instante de la eternidad, objetos distintos y opuestos a los que crea y produce en el ser se fundamenta la esencial contingencia del universo." 19

El obrar causal divino ad extra es en esencia contingente y en él tiene su fundamento la constitución ontológica contingente no sólo de los individuos, sino también del modo en que todos ellos se conectan y estructuran la unidad del universo creado. La apertura sincrónica que la omnipotencia divina mantiene una vez que se ha instaurado cierto orden de cosas hacia las posibilidades no realizadas le permite a Escoto explicar que el creador podría hacer que las cosas tengan un orden distinto al que poseen actualmente. De esta manera, la potentia Dei puede modificar el curso que se espera de los acontecimientos en el mundo natural, como sucede de manera manifiesta en el caso de los milagros.

${ }^{15}$ Lectura, I.39.1-5 § 54 (CS XVII, p. 497).

${ }^{16}$ Vos et al. 1994, p. 125.

${ }^{17}$ Massie 2011, p. 198.

${ }^{18}$ Cfr. Quaestiones super libros Metaphysicorum Aristotelis, IX.15 § 31 (OPh IV, p. 683).

${ }^{19}$ Pérez-Estévez 2004, p. 103.

Diánoia, vol. LXIII, no. 80 (mayo de 2018). 
Para explicar que no hay una contradicción entre la elección de un orden posible y su intervención con otro, el filósofo escocés elabora una interpretación de la potentia absoluta y la potentia ordinata como dos maneras en que puede actuar la misma omnipotencia divina.

La justificación de esta distinción es que todo obrar de un agente libre puede compararse con el cumplimiento de una ley y, así, "puede obrar conforme a aquella ley recta, y entonces según la potencia ordenada [...] y puede obrar lo que está fuera de esa ley o contra ella, y en esto radica la potencia absoluta, que excede a la potencia ordenada". ${ }^{20}$ Cuando un agente carece de la potestad necesaria para instituir una ley, actúa de manera desordenada si sobrepasa la que instituyó rectamente quien tiene la autoridad y el poder de dictarla. Por otro lado, quien esté por encima de la ley puede actuar contra una norma y, sin embargo, hacerlo de manera ordenada siempre que instituya una ley nueva que permita hacer lo que antes no se podía, y esto será posible por su potencia absoluta. Lo último se verifica, por ejemplo, en el caso de un rey respecto de las leyes positivas.

En cuanto legisladora suprema, la omnipotencia divina instituye el orden legal con el que se comporta el universo y la moral, y actúa con potencia ordenada siempre que mantiene el orden establecido. Sin embargo, esto no impide que pueda obrar conforme a otros modos posibles descritos por las leyes a las cuales está sincrónicamente abierta, ya que esa posibilidad se fundamenta en su potencia absoluta y, en tal caso, no lo hará en forma desordena "porque puede instituir otra ley recta según la cual actúe de modo ordenado". ${ }^{21}$ Es por ello que la potentia absoluta divina no es fuente de arbitrariedad en el obrar divino, sino un indicador de la contingencia ontológica que posee el orden creado en todo momento. Para Escoto, la libertad divina no es arbitraria, sino que tiene la capacidad de hacer todo aquello que no incluya contradicción, es decir, lo que se conoce como posible imitación de su esencia.

Es interesante hacer notar que, en los diferentes comentarios a las Sentencias en los que Escoto debe abordar la cuestión de "Si Dios puede hacer un mundo mejor que el que hizo", el filósofo cambia de manera deliberada el asunto por "Si Dios puede hacer las cosas de otra manera a la que ha ordenado que sean". Esto es así porque cualquier orden que Dios quiera es el mejor y puede hacer todos los que sean posibles; incluso una vez que instituye uno determinado, y ya que "todo lo que Dios

${ }^{20}$ Ordinatio, I.44 § 3 (CS VI, pp. 363-364).

${ }^{21}$ Ordinatio, I.44 § 5 (CS VI, p. 365). 
puede hacer es hecho de forma ordenada, se sigue que cualquier orden alternativo que Dios puede otorgar al mundo es igualmente bueno y adecuado. No hay una disposición de cosas más adecuada y mejor posible porque cada disposición, cuando Dios la quiere, es igualmente buena y adecuada." 22

En este contexto, los milagros desafían la explicación del orden natural como algo necesario y estable. Para Escoto, los hechos milagrosos no son una suspensión de la ley establecida por la potentia Dei sobre el mundo físico, sino la prevención del obrar al que una naturaleza está dispuesta ex se, como el fuego a quemar, a causa de una ley nueva dada por la omnipotencia divina a través de la cual dispone otro orden de cosas. Volveré sobre este asunto una vez que desarrolle la relación causal entre el creador y la criatura, para poder determinar si es posible tener certeza en el conocimiento experiencial de un mundo que es contingente en el plano ontológico y en el operativo.

\section{Concurrencia causal entre el creador y las criaturas}

A lo largo de la obra de Escoto encontramos un desarrollo fructífero de la teoría causal, la cual se emplea como herramienta para resolver diversas cuestiones teológicas y filosóficas. Si queremos resumir su pensamiento al respecto, debemos empezar por el orden esencial que propone en el Tractatus de primo principio entendido "en su sentido corriente, en cuanto orden es una relación de comparación mutua entre lo anterior y lo posterior; es decir, en cuanto lo que está ordenado queda suficientemente dividido en anterior y posterior". ${ }^{23}$ El par disyuntivo de propiedades trascendentales anterior-posterior permite distinguir (I) el orden de eminencia y (II) el de dependencia entre los entes. Según esto, lo posterior es lo que depende de lo anterior para ser y lo anterior como "primero en naturaleza y esencia es lo que puede existir sin lo posterior, no viceversa". ${ }^{24} \mathrm{El}$ orden esencial de dependencia representa un orden causal y puede, a su vez, dividirse en (II.A) dependencia del efecto a su causa y (II.B) entre efectos de una misma causa. ${ }^{25}$ En el Tractatus

${ }^{22}$ Pini 2009, p. 295.

${ }^{23}$ J. Duns Escoto, Tratado acerca del primer principio, I § 3 (Alluntis 1989, p. 49).

${ }^{24}$ J. Duns Escoto, Tratado acerca del primer principio, I § 4 (Alluntis 1989, p. 49).

${ }^{25}$ Como esta división se refiere a la dependencia de un efecto que, por naturaleza, es posterior a otro efecto de la misma causa, no es en sentido estricto un orden de dependencia causal aunque podemos incluirlo per extentionem, ya que tiene su origen por una causa. Para una justificación de la etiqueta "no causal", véase King 2003, p. 39.

Diánoia, vol. LXIII, no. 80 (mayo de 2018). 
indica una nueva subdivisión, la famosa enumeración de (II.A.i) las cuatro causas como especies del (II.A) orden esencial de dependencia del efecto a su causa.

Por otro lado, en relación con el modo de causar, Escoto afirma:

debe saberse que una cosa es hablar de causas per se y per accidens, y otra es hablar de causas per se ya sean ordenadas esencial o accidentalmente. Pues en el primer caso hay sólo comparación de una cosa a otra, es decir, de la causa con lo causado: es causa per se la que causa según su propia naturaleza y no según algo accidental suyo, y causa per accidens lo contrario; en el segundo caso hay una comparación de dos causas entre sí, en la medida en que a partir de ellas algo es causado. ${ }^{26}$

En este caso, Escoto nos presenta dos divisiones: (1) causas totales, que actúan (1.A) per se o según su naturaleza ("el constructor que construye") y las que lo hacen (1.B) per accidens o según algo que no pertenece propiamente a su naturaleza ("Policleto construye"). En la otra división encontramos las (2) causas parciales, ya sean (2.A) esencialmente ordenadas o (2.B) accidentalmente ordenadas (dos que tiran de una misma carga). Estas causas parciales se diferencian porque cuando "hay muchas causas concurrentes respecto de un mismo efecto, o concurren con un cierto orden, puesto que son causas esencialmente ordenadas [...] o concurren no con un orden sino en pie de igualdad". ${ }^{27}$

A su vez, el Doctor Sutil profundiza sobre las causas (2.A) esencialmente ordenadas, y diferencia dos modos en los que pueden concurrir varias causas:

(2.A.i) la causa superior mueve a la inferior, y la inferior no tiene la causalidad respecto del efecto por producir sino por la causa superior, de modo que no mueve sino porque es movida por la causa superior [...] (2.A.ii) una es más perfecta en el causar que la otra, que es imperfecta, pero la perfecta no otorga la causalidad a la causa imperfecta para actuar [...] por lo que una no es causa de la causalidad de la otra, sino que sólo es más perfecta y la otra imperfecta. ${ }^{28}$

El primer modo (2.A.i) puede llamarse orden esencial de subordinación, ${ }^{29}$ y el segundo (2.A.ii) orden esencial de conjunción (según este

${ }^{26}$ Ordinatio, I.2.1.1-2 § 47 (CS II, pp. 153-154).

${ }^{27}$ Lectura, I.3.3.2 § 366 (CS XVI, p. 367). Cfr. también Ordinatio, I.3.3.2 § 496 (CS III, p. 293).

${ }^{28}$ Ordinatio, I.3.3.2 § 367 (CS XVI, p. 367).

${ }^{29}$ Como se verá más adelante, el orden de subordinación es entre creador y criatura; cfr. Frank 1992, p. 154; Guzmán Manzano 1990, p. 298. 
último orden, afirma Escoto, intelecto y objeto concurren como causas del acto intelectivo actual).

Por último, en sus comentarios sobre el tema de los sacramentos, desarrolla la división de la subordinación de las causas teniendo en cuenta si son principios activos o no, si pueden alcanzar por su propia forma el término de la acción o no y, por último, si la forma por la que actúan se posee in esse quieto o in fieri. Los modos que resultan son: (2.A.i.a) causa segunda, (2.A.i.b) causa instrumental dispositiva, (2.A.i.c) causa instrumental recibida, (2.A.i.d) causa instrumental parte-todo, y (2.A.i.e) causa instrumental artificial. ${ }^{30}$ De acuerdo con los criterios anteriores, la (2.A.i.a) causa segunda es un principio activo que puede alcanzar el término de la acción del agente principal por su propia forma, que posee in esse quieto. La (2.A.i.b) causa instrumental dispositiva también es activa y actúa por una forma que posee in esse quieto y que dispone un estado de cosas para que la causa principal alcance el efecto. La (2.A.i.c) causa instrumental recibida alcanza el término de la causa principal, pero su forma activa no se tiene in esse quieto, sino que se recibe in fieri, de tal manera que mientras que permanece la subordinación su forma está activada y alcanza por sí el término de la acción. En la (2.A.i.d) causa instrumental del tipo parte-todo, las partes son formas activas que el todo recibe por participación, pero para alcanzar el término no sigue su propia naturaleza, sino la de las partes que se encuentran unidas en el todo. El quinto tipo es la (2.A.i.e) causalidad artificial, específica de los instrumentos fabricados por el ser humano, que no son formas activas y, por lo tanto, no pueden incidir en forma directa en el término de la acción; además, no tienen una intencionalidad propia.

Para Escoto el creador y las criaturas se relacionan como causa primera y causa segunda, ya que "alguna criatura es 'activa' porque recibe totalmente su causalidad de Dios respecto del propio efecto. Pero esto no repugna a que una criatura (como el fuego) tenga la causalidad total hacia algún efecto (como el calor)". ${ }^{31}$ Lo distintivo de las causas segundas como principios activos es que pueden alcanzar por su propia naturaleza el término de la acción, y así sucede con los agentes físicos, ya que han sido creados con una naturaleza específica que los hace poseer ciertas disposiciones para actuar de una manera determinada. Los entes del mundo natural qua eficientes son principios activos que causan según el modo de naturaleza, de tal manera que actualizarán

${ }^{30}$ Cfr. Ordinatio, IV.6.3.1 §§ 113-129 (CS XI, pp. 330-334). Tomo los nombres de Cross 2008, p. 287.

${ }^{31}$ Lectura, II.34-37.1-5 § 126 (CS XIX, p. 358).

Diánoia, vol. LXIII, no. 80 (mayo de 2018). 
sus disposiciones siempre que el objeto se haga presente y no sean impedidos por algo distinto de ellos.

Esta subordinación de las causas segundas a la causa primera tiene una raíz doble, ontológica y operativa, puesto que el ser de las criaturas depende de manera continua de la conservación que proporciona el creador y, por medio de esto, sus operaciones están supeditadas a la existencia del sujeto activo. Sin embargo, esta dependencia no elimina la causalidad real, propia y específica, con la cual han sido dotadas las causas segundas, porque esto implicaría negar la naturaleza activa de los agentes al reducir toda la actividad del mundo natural a un acto inmediato de la voluntad divina o limitar la capacidad de los entes físicos al convertirlos en meros instrumentos. Si bien "el fuego puede quemar la estopa por la influencia general de Dios", 32 se puede decir que el fuego es la causa principal porque "obra por su propia e intrínseca forma, aunque — actuando por ella— esté subordinada a la causa agente superior". ${ }^{33}$

Como indiqué antes, el obrar divino no es arbitrario ni contradictorio; por eso, una vez que se crean los entes físicos según diversas naturalezas y se conservan en el ser, cada uno de ellos actualizará su actividad causal de acuerdo con sus propias aptitudes y en la medida en que la relación con los demás seres creados lo haga posible de acuerdo con la disposición que el creador, por su potentia ordinata, ha establecido. "De todas maneras, Dios no puede organizar las cosas de tal modo que ciertas disposiciones no pertenezcan a ciertas esencias, puesto que tales disposiciones son realmente idénticas a las esencias a las que pertenecen." 34 La naturaleza de cada ente ha sido determinada para existir por la voluntad divina según la idea producida por su intelecto, de tal manera que el obrar providente "respeta" esas naturalezas y las rationes por las cuales actúan. "Por eso, es evidente que no hay una influencia nueva de la causa primera sobre la causa segunda propiamente, que sea creación de algo inherente a la causa segunda, cuando actúan al mismo tiempo; sino que esa influencia es un orden determinado de esas causas para obrar un efecto común." 35

Es importante mostrar hasta qué punto el Doctor Sutil sostiene que la acción divina respeta el orden querido y determinado para cada naturaleza, porque allí radica la clave para comprender la capacidad de

\footnotetext{
${ }^{32}$ Lectura, I.3.1.3 § 144 (CS XVI, p. 281).

${ }^{33}$ Ordinatio, IV.1.1.1 § 119 (CS XI, p. 43).

${ }^{34}$ Pini 2009, p. 291.

${ }^{35}$ Ordinatio, IV.1.1.1 § 170 (CS XI, p. 60).
} 
alcanzar la certeza que puede adquirir nuestro conocimiento experiencial y por qué lo que se considera milagro no es un obrar contra las leyes de la naturaleza. ${ }^{36}$ En efecto, al afirmar por su creencia cristiana ${ }^{37}$ que Dios, en cuanto agente perfectísimo, puede actuar de manera inmediata para crear cualquier cosa posible, admite que en la materia creada hay una potencia obediencial o sobrenatural. Ésta consiste en la "sujeción respecto de un agente que es capaz de hacer [a otro] obediente en lo que él quiere", ${ }^{38}$ y por ello la materia tiene potencialidad para recibir cualquier forma que la omnipotencia divina desee imprimir inmediatamente en ella. Si bien la acción divina es perfecta y puede actuar de manera inmediata en el mundo creado, nunca lo hace de manera que rompa las aptitudes establecidas para cada naturaleza "pues aunque Dios podría absolutamente crear el frío, sin embargo, no podría crearlo por medio del calor, de modo que el calor según su propio orden sea principio activo respecto del frío (o, en cualquier otro ejemplo, donde habría tal repugnancia en el obrar)". 39

Ahora bien, ¿cómo puede ser compatible este "respeto" de la acción divina sobre las naturalezas creadas con la manifestación de los milagros, es decir, de una intervención especial en el mundo físico por la que los seres no se comportan de acuerdo con su naturaleza?

Para responder a este planteamiento, debemos recordar que los entes físicos actúan según el modo de naturaleza, lo que para Escoto significa que su acción causal es necesaria siempre que no sea impedida desde afuera; o, si queremos expresarlo de una manera más precisa, su acción causal está determinada a producirse cuando un objeto se hace presente y se prolongará en la medida en que no haya un impedimento extrínseco. Cada ser físico posee ciertas disposiciones intrínsecas a partir de las que puede desarrollar su naturaleza específica, y cada individuo está inclinado a actuar por ellas, en la medida en que esté de su parte, según una manera determinada. Esas disposiciones o inclinaciones de cada ente físico señalan una "dependencia aptitudinal" 40

${ }^{36}$ Cfr. Todisco 1984, p. 311: "A todo esto se suman las reservas que de vez en cuando Escoto insinúa cuando cree posible la consecución de la causa del fenómeno. Es decir, la posibilidad de que la causa no sea impedida a obrar, que no haya nada 'contra natura'."

${ }^{37}$ Cfr. Reportatio parisiensis examinata, I-A.42.1-2 § 13 (Wolter Bychkov II, p. 512): "Por el primer modo [i.e. tener omnipotencia hacia todos los posibles de manera inmediata] los católicos aceptan la omnipotencia [divina], la cual no puede ser probada por medio de la razón natural, sino que sólo [puede ser] creída".

${ }^{38}$ Quaestiones super libros Metaphysicorum Aristotelis, IX.12 § 11 (OPh IV, p. 614).

${ }^{39}$ Ordinatio, IV.1.1.1 § 121 (CS XI, p. 44).

${ }^{40}$ Cfr. Ordinatio, III.1.1.1 § 45 (CS IX, p. 20): "A esto llamo [dependencia] apti-

Diánoia, vol. LXIII, no. 80 (mayo de 2018). 
respecto de las acciones por la que consiguen los fines adecuados a su naturaleza, ${ }^{41}$ es decir, la tensión que hay entre acto primero y acto segundo.

Además, cada naturaleza actualiza sus aptitudes gracias a la interacción con otros entes; por ello, toda actividad causal en el mundo natural depende de la organización con que se encuentran interrelacionadas las cosas. De hecho, la lluvia "puede darse por el concurso de unas estrellas, pero puede suceder que no llueva cuando otras concurren, porque quizá algo más que se les suma lo impide". ${ }^{42}$ En este sentido, se entiende que una organización determinada del universo puede impedir la actualización de algunas aptitudes de los entes, pero si esas mismas naturalezas estuvieran dispuestas de otra manera podrían activar su capacidad causal. Según Escoto, esto último es posible para la omnipotencia divina, como he precisado en la sección anterior.

Por lo tanto, los milagros no implican una acción contra la naturaleza de las cosas, sino una nueva disposición específica por la que algún ente físico se ve impedido de actualizar las aptitudes propias de su naturaleza. Esos diferentes modos de organizar las cosas responden a un querer eterno divino, por el que se realizarán en un momento determinado de la historia según lo fija su voluntad y por medio de una ley establecida según la potentia ordinata.

Como comienza a hacerse evidente, el modo de causar necesario y el natural no son idénticos, ya que "algo natural puede actuar de manera contingente, porque puede ser impedido". ${ }^{43}$ En la Reportatio parisiensis Escoto examina dos modos de necesidad, a saber, la inmutabilidad y la inevitabilidad (a los que contrapone la contingencia de mutabilidad y de evitabilidad). El primer tipo de necesidad es propio de la esencia divina, pero a los entes físicos les corresponde el segundo porque la necesidad ex se de su actividad puede prevenirse extrínsecamente. ${ }^{44}$

tudinal, a la que siempre en cuanto es de sí sería en acto, del modo como lo pesado tiene aptitud para ir hacia abajo, donde siempre estaría en cuanto le corresponde de sí en la medida en que no sea impedido".

${ }^{41}$ Cfr. Quaestiones quodlibetales, 19.3 § 67 (Alluntis 1968, p. 689): "Porque aquella [aptitud] no es propiamente sino hacia una forma perfectiva por naturaleza".

${ }^{42}$ Lectura, II.14.4 § 36 (CS XIX, p. 126).

${ }^{43}$ Quaestiones quodlibetales, 16.2 § 34 (Alluntis 1968, p. 597).

${ }^{44}$ Cfr. Reportatio parisiensis examinata, I-A.39-40.1-3 § 25 (Wolter Bychkov II, pp. 471-472): "La necesidad de inmutabilidad es aquella que no puede darse de otra manera, del modo como Dios es necesario. Por otro lado, la necesidad de inevitabilidad es aquella por la que un suceso de alguna cosa futura se dice inevitable, aunque en sí no sea ni inmutable ni necesario, como que el Sol saldrá mañana es necesario con necesidad de inevitabilidad, y otros movimientos naturales son 
Por lo tanto, para precisar lo específico de cada noción se puede decir que "obrar naturalmente es obrar sin autodeterminación y obrar necesariamente es hacerlo inevitablemente". ${ }^{45}$

Con respecto al tema de la subordinación, Escoto afirma que tanto la causalidad primera del creador respecto de las criaturas como la influencia general que mantiene en todo momento sobre las causas segundas son contingentes. Por ello, toda actividad natural de los entes físicos depende de la causa primera, de tal manera que su causalidad será necesaria si, y sólo si, no es impedida especialmente por ella. De otra manera, se puede decir que una naturaleza causará todo lo que esté de su parte en la medida en que la causa primera coopere con ella y mientras tal cooperación se mantenga. En consecuencia, todo efecto que un ente físico cause de manera necesaria se subordinará sobre todo a la actividad contingente de la causa primera, y "es producto de una doble acción causativa conjugada: la divina, que es contingente, y la natural, que es necesaria. El efecto, aunque necesario para la causa segunda, se produce en definitiva contingentemente". ${ }^{46}$

En este contexto, la contingencia ontológica y causal característica del universo físico tal como la plantea Duns Escoto parece comprometer cualquier posibilidad de certeza que nuestro conocimiento experiencial pueda alcanzar. En la siguiente sección determinaré el alcance de esta objeción.

\section{La certeza en el conocimiento experiencial del mundo físico}

C. Ledsham ${ }^{47}$ propuso hace poco una interpretación de la potentia Dei y su concurso en el mundo natural en la cual intenta mostrar que existe cierta incoherencia en el pensamiento de Duns Escoto respecto de la certeza que puede alcanzar nuestro conocimiento experiencial. Afirma que "hay una inconsistencia en el análisis que hace Escoto de la contingencia en lo que se refiere a la ley física, por un lado, y a la ley natural ética, por el otro". ${ }^{48}$ Sobre la ley moral, el Doctor Sutil sostiene que los mandamientos de la primera tabla son necesarios porque se refieren a Dios, pero algunos de los que se refieren al prójimo, en la segunda tabla, pueden ser dispensables y, de hecho, lo han sido en algún momento de

necesarios por lo mismo y, sin embargo, pueden darse de otra manera, por lo que no son absolutamente necesarios ni inmutables."

${ }^{45}$ Solaguren 1968, p. 307.

${ }^{46}$ Solaguren 1968, p. 327.

${ }^{47}$ Cfr. Ledsham 2010, pp. 557-575.

${ }^{48}$ Ledsham 2010, p. 568. 
la historia de la salvación. Sin embargo, estos preceptos "dispensables" pertenecen a la ley natural large loquendo, ya que no son necesarios pero están en consonancia con aquellos que sí lo son. Ahondar en esta cuestión me alejaría de mis propósitos; baste decir que la dispensa de esos preceptos es consistente con el obrar contingente divino según lo entiende Escoto. Esta situación no parece repetirse en la explicación que propone sobre el conocimiento experiencial, cuando afirma, por ejemplo, que "aunque no se tenga la experiencia de todos los casos singulares, sino de muchos, ni siempre, sino muchas veces, de todos modos el experto aprehende [novit] infaliblemente que es así siempre y en todos los casos". 49

Si suponemos que la causa primera actúa sobre todo orden creado, natural y moral, de modo contingente, y que la potentia Dei está abierta sincrónicamente a infinitos órdenes posibles, la afirmación sobre la existencia de un conocimiento experiencial infalible no parece hacer justicia a la ontología del mundo físico antes planteada, porque no es posible llegar a un conocimiento infalible de lo contingente. Además, se debe tener en cuenta que la creencia en los milagros muestra la conciencia de Escoto en la contingencia de la causalidad, en el mundo natural, porque el modo de obrar por naturaleza no implica la inevitabilidad, sino la incapacidad de autodeterminación, y, así, todo agente físico está condicionado por elementos extrínsecos para actuar. En este contexto, parece contradictorio afirmar que podemos alcanzar una certeza sobre la ley física "el fuego quema" si, de hecho, en ocasiones observamos que no lo hace.

Ledsham resume sus críticas de la siguiente manera:

Primero, podemos sostener que la condición de Escoto para el principio de causalidad natural, por la que excluye acciones causadas de manera libre, es una suerte de prestidigitación. Todo en la naturaleza es dependiente, en última instancia, de una acción causada libremente, de tal manera que no podemos hacer en realidad la distinción entre causas naturales y libres.

Una segunda manera de encuadrar el problema es leer a Escoto como si pretendiera simplemente que su solución al problema de la inducción alcance un orden físico seguro y necesario para la naturaleza, con la excepción superflua en términos teóricos de las causas queridas libremente.

Una tercera manera de proceder es explorar las formas de conocimiento científico del mundo natural que, según Escoto, podemos obtener como resultado del principio de causalidad natural. En su solución a la inducción, Escoto se refiere a un mero conocimiento de correlaciones contingentes

${ }^{49}$ Ordinatio, I.3.1.4 § 235 (CS III, p. 141).

Diánoia, vol. LXIII, no. 80 (mayo de 2018). 
(e indica que una causa natural tiene una aptitud para producir un efecto) como la forma más baja de scientia (de una causa quia), aunque una scientia que es, con todo, indubitable. ${ }^{50}$

A continuación analizaré los diferentes enfoques con los cuales el Doctor Sutil desarrolla el tema del conocimiento experiencial, para luego ofrecer a la objeción planteada una respuesta ad mentem Scoti.

Al comienzo de la Metafísica, Aristóteles sostiene que "nace el arte cuando de muchas observaciones experimentales surge una noción universal sobre los casos semejantes". ${ }^{51}$ Por ello, denomina "sabio" a quien conoce las causas de las cosas y posee su arte; por otro lado, el "experto" es aquel que no conoce el porqué sino el qué, basado en el conocimiento de las cosas singulares. En su comentario a estas ideas, Escoto añade que es posible, por medio del método de la división, llegar a conocer desde un efecto sensible cuál es su causa. ${ }^{52} \mathrm{El}$ análisis deberá seguir la siguiente metodología: hay que identificar primero los factores que se dan juntos en la consecución de un efecto y luego separarlos para observar si el resultado es aún el mismo. A continuación, se deberán dejar de lado aquellos factores que no se encuentran aunque se consiga el efecto de igual manera. Por último, se deberá observar cuáles de los factores restantes son anteriores por naturaleza para identificarlos como las causas.

Con todo, el Doctor Sutil introduce una instantia en la que desarrolla un contraargumento basado en la falacia de afirmación del consecuente. ${ }^{53}$ Como respuesta, hace notar que por el método de división se puede reconocer que al menos una causa determinada es necesaria para conseguir un efecto específico. Asimismo, afirma que el "experto" posee su conocimiento por el análisis de los efectos, quia est, pero, para lograr una demostración propter quid, no basta con saber cuál es la causa, sino que se requiere saber por qué la causa es la causa. Sin embargo, "el experto, al carecer de demostración, sabe quia est con certeza", ${ }^{54}$ y esto es posible gracias a un principio por el que el intelecto capta de manera directa el obrar uniforme y ordenado de la naturaleza: "la naturaleza obra [de la misma manera] en la mayoría de los casos, si

${ }^{50}$ Ledsham 2010, p. 571.

${ }^{51}$ Aristóteles, Metafísica, 981a 5-7 [trad. A. García Yebra].

${ }^{52}$ Cfr. Quaestiones super libros Metaphysicorum Aristotelis, I.4 § 70 (OPh III, p. 116). Se sugiere consultar el cuadro esquemático en Vos 2006, pp. 312-313.

${ }^{53}$ Cfr. Quaestiones super libros Metaphysicorum Aristotelis, I.4 §§ 71-73 (OPh III, pp. 116-117).

${ }^{54}$ Quaestiones super libros Metaphysicorum Aristotelis, I.4 § 79 (OPh III, p. 118).

Diánoia, vol. LXIII, no. 80 (mayo de 2018). 
no es impedida", ${ }^{5}$ lo que Ledsham denomina "principio de causalidad natural". Escoto no aclara más y da por resuelto el asunto.

Ahora bien, en diferentes pasajes de su obra sostiene que toda ciencia debe proporcionar un conocimiento que sea cierto, necesario, evidente y resultado de un razonamiento silogístico. ${ }^{56}$ Esta última característica no es aplicable aún a lo que desarrolla sobre la inducción y su actividad sobre el intelecto. Aborda esta cuestión en los comentarios a las Sentencias, cuando retoma la explicación sobre el conocimiento experiencial como respuesta a la teoría de la iluminación especial según la propuesta que formula Enrique de Gante. En este caso, negará tal necesidad en el intelecto humano viatoris y sostendrá la posibilidad de certeza por el conocimiento natural de los primeros principios, de la experiencia y de nuestros propios actos.

En la Lectura afirma que podemos alcanzar certeza tanto de una conclusión como de un principio a partir del conocimiento experiencial. La experiencia nos brinda una conclusión que obtenemos mediante la observación de un efecto que sucede con frecuencia, como por ejemplo "la Luna se eclipsa" o "esta hierba cura tal enfermedad". Junto con esto, vuelve a formular el principio causal y agrega una conexión entre la frecuencia de los efectos y su causa natural, "aquello que sucede en la mayoría de los casos es un efecto natural y tiene una causa naturalmente ordenada a tal efecto". ${ }^{57}$ Este principio per se notum garantiza el paso de un conocimiento cierto sobre casos singulares in pluribus a todos in omnibus. Esta metodología tiene un fundamento epistemológico en la doctrina, adoptada por Escoto, de la natura communis, según la cual toda esencia es indiferente a la universalidad e individualidad, ${ }^{58}$ modos que adoptará si el intelecto la conoce como objeto de su acto o si la contrae una formalidad que la determina para ser haec. De tal manera, al conocer por experiencia la naturaleza de un ente físico singular y

${ }^{55}$ Cfr. Quaestiones super libros Metaphysicorum Aristotelis, I.4 $\S 68$ (OPh III, pp. 115-116).

${ }^{56}$ Sobre el desarrollo cronológico y la extensión del concepto aristotélico de scientia en Escoto, véase Marrone 2009, pp. 383-400.

${ }^{57}$ Lectura, I.3.1.3 § 177 (CS XVI, p. 294).

${ }^{58}$ Cfr. Ordinatio, II.3.1.1 § 34 (CS VII, p. 404): "Y como según aquella existencia [en el intelecto] no es la naturaleza de sí universal, sino que la universalidad es un accidente de la naturaleza de acuerdo con su primera razón, según que es objeto, así también en las cosas extramentales, en las que la naturaleza se encuentra con la singularidad, no está aquella naturaleza de sí determinada a tal singularidad, sino que es anterior por naturaleza a la misma razón que la contrae a esa singularidad, y en cuanto es anterior por naturaleza a lo que la contrae, no le repugna existir sin aquello que la contrae". 
aplicar el principio per se notum de causalidad natural, queda justificado el paso de muchos a todos, aunque sus operaciones se ejecuten con necesidad del tipo de inevitabilidad.

Por otro lado, a partir de una conclusión y aplicando el método de división, se pueden determinar las causas siempre que se eliminen todos los factores que se puede demostrar que no intervienen en el fenómeno. Si tomamos el ejemplo del eclipse lunar, el intelecto debe aplicar el método a las respuestas posibles, y aclarar si la Luna posee en sí misma luz y por un defecto propio se eclipsa o, por el contrario, la luz es alcanzada por la interposición de otro cuerpo que produce el eclipse en la Luna. ${ }^{59}$ Es interesante hacer notar que, sin hacerlo explícito, Escoto recurre aquí a hipótesis que puedan explicar el hecho y, por lo tanto, a un razonamiento de tipo deductivo ${ }^{60}$ por el que alcanzará el conocimiento propter quid de la investigación en cuestión.

En la Ordinatio, Escoto vuelve a precisar el principio causal y aclara que se aplica a causas no libres y que el intelecto lo conoce aun cuando los sentidos yerran: "aquello que sucede en la mayoría de los casos por una causa no libre es un efecto natural de aquella causa". ${ }^{61}$ La idea detrás de la aclaración es que puede haber contingencia en la causa, que es lo característico del modo de la voluntad, o en el efecto, porque los entes que actúan según el modo de la naturaleza se pueden ver impedidos por algo extrínseco que supera la virtus de su naturaleza causal ya que el modo de naturaleza está determinado a obrar de la misma manera cuando se dan las condiciones adecuadas. A continuación, el filósofo explicita dos modos posibles en los que puede derivar la investigación científica. Esta aclaración no aparece con precisión en sus otras obras y constituye un pensamiento de madurez sobre el tema.

Una primera posibilidad es que a partir de una conclusión observada con frecuencia, conocimiento quia est, se llega a la comprensión del principio que actúa en ese caso y, a partir de él, se deduce, por el método de división, la causa real. Mediante la aplicación del principio causal, Escoto propone un nuevo método de división por vía del acuerdo entre diferentes instancias de la experiencia y la observación de los factores que intervienen en cada una, siempre que se consiga el mismo efecto. De esta manera, la atención se centra en descubrir qué factor está presente en cada una de las instancias, porque no se le puede atribuir causalidad alguna a un factor que unas veces está presente y otras no

${ }^{59}$ Cfr. Lectura, I.3.1.3 § 178 (CS XVI, p. 295).

${ }^{60}$ Cfr. Vos 2006, p. 316.

${ }^{61}$ Ordinatio, I.3.1.4 § 235 (CS III, p. 142).

Diánoia, vol. LXIII, no. 80 (mayo de 2018). 
lo está. El que se encuentre en todas las ocasiones será la causa natural del efecto. ${ }^{62}$ El Doctor Sutil recurre de nuevo al ejemplo del eclipse. Vemos con frecuencia que la Luna se eclipsa, y ésta es una conclusión de experiencia; pero, para conocer su causa, debemos alcanzar el principio al que corresponde su explicación. El intelecto lo descubre como proposición evidente por sus términos, a saber, que "todo cuerpo opaco interpuesto entre uno luminoso y otro iluminado, impide la transmisión de la luz". ${ }^{63}$ Luego se aplica el método de división por el cual se llega a conocer que la Tierra es un cuerpo opaco, interpuesto entre el Sol y la Luna. Este conocimiento posee un importante grado de certeza, certissime, ya que se obtiene por medio de una demostración propter quid desde el dato de experiencia y se establece una relación causal absoluta, ya que puede atribuirse no sólo a muchos, sino a todos los casos.

Sin embargo, puede suceder que a partir de la experiencia no lleguemos al conocimiento del principio evidente que explica el fenómeno, sino que sólo conozcamos un principio que surge mediante la generalización de los casos en los que se observan unidos ciertos términos. Incluso si se aplica el principio empírico de uniformidad de la causalidad natural, tampoco se podrá tener un conocimiento infalible si no se produce por vía de la demostración la explicación de por qué esos términos están unidos. El ejemplo que propone Escoto es el siguiente: "Esta hierba de tal especie es cálida". Puede suceder que un médico utilice esa hierba porque hace bien a la salud, pero no conocería la relación que hay entre la enfermad y la hierba ni entre la calidez de la hierba y su efecto, e incluso podría darse que esa misma hierba también sea saludable a una temperatura menor. Escoto explica con el ejemplo que este tipo de generalizaciones sólo nos lleva a conocer la unión aptitudinal, pero no la unión real de los términos, ya que éstos podrían encontrarse incluso separados, sin que esto implique una contradicción. ${ }^{64}$ En ese caso, tendremos un conocimiento cierto, pero contingente, asegurado por el principio de uniformidad que el creador mantiene en el mundo natural. Sin embargo, Escoto afirma que es el grado más bajo de conocimiento científico.

Como se desprende del resumen precedente, la intención de Escoto es fundar el conocimiento experiencial en principios universales que aseguren la certeza que se deriva del conocimiento de muchos casos particulares, aunque reconoce la dificultad de alcanzar una verdadera

${ }^{62}$ Cfr. el cuadro esquemático en Vos 2006, p. 317.

${ }^{63}$ Ordinatio, I.3.1.4 § 236 (CS III, p. 143).

${ }^{64}$ Cfr. Ordinatio, I.3.1.4 § 237 (CS III, pp. 143-144). 
ciencia cuando nuestro intelecto sólo puede descubrir la unión de hecho entre una naturaleza y su accidente. De cualquier forma, también está consciente de que toda causalidad natural es, en última instancia, contingente porque depende de la actividad divina libre y reconoce esto de manera explícita cuando dice:

[T] oda causa segunda depende de su relación con la causa primera respecto del efecto; de modo similar, ninguna causa segunda causa sino concausando su efecto junto con la causa primera [...] y así la necesidad es secundum quid —en cuanto está de su parte- y no simpliciter: como el fuego, en cuanto está de su parte, no puede no calentar; sin embargo, puede absolutamente no calentar cuando Dios no coopera, como sucedió con los tres jóvenes en el horno. ${ }^{65}$

Ahora bien, a mi juicio, Duns Escoto no es inconsistente en su planteamiento de alcanzar la certeza en el conocimiento experiencial, porque esa seguridad puede adquirirse siempre que nuestro intelecto, con ocasión del conocimiento sensible, alcance a reconocer que ciertas aptitudes pertenecen a una naturaleza aunque no se actualicen en todas las circunstancias por causa de factores extrínsecos, ya sea por la actividad causal de otras naturalezas o por la de la misma potentia Dei. Como se vio en la primera sección, la voluntad divina no puede cambiar las aptitudes propias de una naturaleza concebida por el intelecto divino como modo imitable de su esencia, sino sólo qué naturalezas existirán. Por ello, la certeza del conocimiento experiencial encuentra su fundamento en la capacidad de nuestro intelecto para conocer las esencias de las cosas según las concibió la mente divina, como ideas o reglas eternas. ${ }^{66}$ En efecto, la realidad ontológica de los entes físicos es contingente respecto de la existencia, pero esto no quiere decir que la estructura interna de sus naturalezas y aptitudes sean modificables, sino que, por el contrario, responden al modo en que han sido producidas según las reglas eternas a partir de las cuales la omnipotencia divina determinó que algunas existieran. Con todo esto, podemos ahora responder los planteamientos de Ledsham.

Respecto del primero, la distinción entre causa natural y libre se justifica en el pensamiento de Escoto porque el criterio responde al modo

${ }^{65}$ Ordinatio, I.8.2.1 § 306 (CS IV, p. 328). Cfr. Lectura, I.8.2.1 §§ 284-285 (CS XVI, pp. 107-108), Quaestiones super libros Metaphysicorum Aristotelis, V.3 § 27 (OPh III, pp. 435-436).

${ }^{66}$ Cfr. Lectura, I.3.1.3 § 189 (CS XVI, p. 301): "La verdad inefable y cierta puede conocerse por nosotros en la luz eterna". 
de obrar que le compete a un ente o facultad ex se. El principio de causalidad natural es la formulación explícita de que los entes físicos tienen una naturaleza por la que obran de una manera determinada en cuanto a lo que les compete. Por ello, incluso en un mismo ser, se puede encontrar una facultad que actúa según el modo de naturaleza, el intelecto, y otra que lo hace libremente, la voluntad. La dependencia operativa de la causa segunda hacia la causa primera no elimina la naturaleza ontológica de los entes físicos, sino que los condiciona respecto de sus posibilidades operativas.

A la segunda objeción puede responderse que, si bien toda causalidad en el mundo físico depende de la causa primera, la idea de subordinación de Escoto respeta la estructura de la naturaleza de cada ente y sus aptitudes concretas. ${ }^{67}$ Cuando esto no sucede, como cuando el fuego no quema, es porque cierta actividad se previene aunque no se destruya. La omnipotencia divina no afecta la realidad ontológica de cada naturaleza sino para darle existencia; por ello, el conocimiento absolutamente infalible es el que podemos adquirir sobre las naturalezas de los entes físicos, iniciado por los sentidos pero asegurado por la luz de la inteligencia cuando abstrae la naturaleza de las cosas que participan en las ideas divinas.

Sobre la tercera objeción, estoy de acuerdo en que la experiencia nos ofrece un grado de ciencia muy bajo, aunque indubitable, en especial porque sólo alcanza a formular generalizaciones empíricas. Pero para comprender correctamente esta idea podemos traer a colación un pasaje que Ledsham no tiene en cuenta, y en el cual Escoto dice:

[A]cepto que no hay ciencia absolutamente necesaria, sino sólo en la mayoría de los casos. Pues, a una cosa se le permite, la mayoría de las veces, obrar según sus movimientos [propios] y un orden dispuesto para ella. Sin embargo, algunas veces al permitírsele aquel orden, obra según otro orden. Es evidente [en el caso] de Josué acerca de la paralización del Sol y los jóvenes en el fuego [...]. Ahora bien, que [las cosas] sean por naturaleza aptas para tales movimientos, es necesario y conocido demostrativamente. Pero que sus movimientos sean de otro modo [...] esto es conocido contingentemente, y por eso no puede haber conocimiento demostrativo. ${ }^{68}$

${ }^{67}$ Cfr. Todisco 1984, p. 316: "La disponibilidad del sujeto potencial se subordina sólo a su capacidad receptiva constituida según su naturaleza particular, pero cuyos efectos producidos según el curso ordinario no son su expresión completa".

${ }^{68}$ Reportatio parisiensis examinata, I-A.44.1 § 17 (Wolter Bychkov II, p. 536). Cfr. los ejemplos bíblicos en Josué 10:13 y Daniel 3:6-26. 
La clave está en que la ausencia de duda apunta a que es posible alcanzar una certeza de las aptitudes esenciales de un ente físico que, con ocasión del conocimiento experiencial y junto con el principio de causalidad natural, nuestro intelecto logra captar por tener el poder de conocer las naturalezas de las cosas. Esa seguridad tiene su fundamento en las ideas divinas, porque cada ser creado participa de ellas y la voluntad divina no puede por su potentia absoluta modificarlas, sino sólo determinarlas a existir junto con otras. Así es que los entes físicos actuarán conforme a sus naturalezas siempre que puedan, y nuestro conocimiento experiencial será la fuente de información para alcanzar un conocimiento cierto de ellas por la capacidad que tenemos como seres intelectuales.

Por último, no quiero dejar de subrayar que Escoto utiliza un concepto de scientia más amplio que el que presenta Aristóteles en los Analíticos posteriores para poder justificar de qué manera la teología es posible si versa sobre cuestiones necesarias pero contingentes. Es posible afirmar la certeza del conocimiento, aunque sea sobre una realidad contingente, porque no es "la necesidad del objeto lo que hace científica a una investigación, sino el rigor con el que el sujeto procede en el acercamiento a los hechos y en el establecimiento de las relaciones causales" ${ }^{69}$ En efecto, se puede hablar de un conocimiento científico de lo contingente en un sentido amplio "tomando la palabra ciencia en el sentido usado por el Filósofo en IV Ethicorum, es decir, en contraposición a la opinión o conjetura", ${ }^{70}$ y por ello también la determinación de las generalizaciones empíricas puede entrar en la noción de conocimiento científico.

La potentia Dei en el pensamiento de Duns Escoto nunca actúa de modo arbitrario, sino que circunscribe su capacidad a todo lo posible sin dejar por ello de ser omnipotente. Es el intelecto divino quien produce los posibles en cuanto imitaciones de la esencia divina, y a partir de ellos la voluntad divina determina libremente cuáles recibirán la existencia. Cada ente físico posee una naturaleza que participa de una idea divina y por la cual posee ciertas aptitudes para obrar de determinada manera siempre que no se vea impedido desde afuera. La prevención de su obrar puede estar motivada por el ejercicio de la causalidad de otro ente del mundo natural que lo supera, o sobre todo por el ejercicio de la potentia Dei que, como causa primera universal, influye en toda actividad de los seres creados.

69 Todisco 1984, p. 307.

${ }^{70}$ Ordinatio, Prol., 4.1-2 § 212 (CS I, pp. 145-146). 
Sin embargo, la subordinación de las causas segundas no implica que su causalidad logre actualizar aptitudes que no pertenecen a su naturaleza, pues esto implicaría un obrar contradictorio para la potentia Dei. Con todo, la causa primera puede disponer, eternamente, cierto orden de cosas para un momento determinado y otro distinto para un momento posterior. De esta manera, según el orden querido por Dios, las naturalezas de los entes físicos podrán actualizar o no sus aptitudes, siempre en conformidad con las leyes que establece su potentia ordinata. En este sentido, los milagros no representan un rechazo de las leyes de la naturaleza, sino la elección eterna para una ocasión determinada de un nuevo orden físico por el que se ve prevenida la aptitud de ciertas naturalezas.

Por último, la contingencia ontológica del mundo físico creado y la contingencia operativa de los entes en lo que respecta a su causalidad no afectan a la constitución interna por la que cada naturaleza posee aptitudes propias, pues participan de las ideas o reglas eternas de la mente divina. Por ello, Duns Escoto defiende la capacidad de adquirir certeza a partir del conocimiento experiencial, porque éste nos manifiesta las aptitudes que son propias de cada ente. Además, aunque esas aptitudes no se actualicen siempre o nosotros no las conozcamos en todos los casos, el intelecto puede alcanzar un conocimiento seguro de la naturaleza de los entes físicos gracias al principio de causalidad natural, pues la ausencia de un obrar frecuente indica la prevención de una causalidad que es propia.

En definitiva, la objeción de inconsistencia no tiene en cuenta: a) que para el Doctor Sutil las naturalezas de los entes físicos tienen una estructura interna y aptitudes que actualizarán necesariamente siempre que puedan; b) que el conocimiento experiencial es ocasión para que el intelecto aprehenda esas naturalezas conforme han sido pensadas y queridas por Dios, y c) que, a pesar de su omnipotencia, la voluntad divina no puede modificar dichas naturalezas por su acción libre.

\section{BIBLIOGRAFÍA}

Autores antiguos y medievales

Aristóteles, Metafísica, trad. V. García Yebra, Gredos, Madrid, 1970.

Avicena, Liber de philosophia prima sive scientia divina I-IV, ed. S. van Riet, Brill (Avicenna Latinus, III), Leiden, 1977.

Enrique de Gante, Summae quaestionum ordinariarum, J. Badius, París, 1520, 2 vols. [ed. E.M. Buytaert, The Franciscan Institute (Text Series, 5), Nueva York, 1953]. 
Duns Escoto, J.

Alluntis 1968 Cuestiones cuodlibetales, ed. F. Alluntis, Católica (Biblioteca de Autores Cristianos, 277), Madrid, 1968.

Alluntis 1989 Tratado acerca del primer principio, ed. F. Alluntis, Católica (Biblioteca de Autores Cristianos, 503), Madrid, 1989.

CS (Commissio Opera omnia, vol. I, Ordinatio, Prologus, ed. C. Balic et al., Scotistica) I

CS II Typis Polyglottis Vaticanis, Ciudad del Vaticano, 1950.

Opera omnia, vol. II, Ordinatio I, dist. 1-2, ed. C. Balic et al., Typis Polyglottis Vaticanis, Ciudad del Vaticano, 1950.

CS III Opera omnia, vol. III, Ordinatio I, dist. 3, ed. C. Balic et al., Typis Polyglottis Vaticanis, Ciudad del Vaticano, 1954.

CS IV Opera omnia, vol. IV, Ordinatio I, dist. 4-10, ed. C. Balic et al., Typis Polyglottis Vaticanis, Ciudad del Vaticano, 1956.

CS VI Opera omnia, vol. VI, Ordinatio I, dist. 26-48, ed. C. Balic et al., Typis Polyglottis Vaticanis, Ciudad del Vaticano, 1963.

CS VII Opera omnia, vol. VII, Ordinatio II, dist. 1-3, ed. C. Balic et al., Typis Polyglottis Vaticanis, Ciudad del Vaticano, 1973.

CS IX Opera omnia, vol. IX, Ordinatio III, dist. 1-17, ed. B. Hechich, Typis Polyglottis Vaticanis, Ciudad del Vaticano, 2006.

CS XI Opera omnia, vol. XI, Ordinatio IV, dist. 1-7, ed. B. Hechich, Typis Polyglottis Vaticanis, Ciudad del Vaticano, 2008.

CS XVI Opera omnia, vol. XVI, Lectura, Prologus-I, dist. 1-7, ed. C. Bali et al., Typis Polyglottis Vaticanis, Ciudad del Vaticano, 1960.

CS XVII Opera omnia, vol. XVII, Lectura I, dist. 8-45, ed. C. Bali et al., Typis Polyglottis Vaticanis, Ciudad del Vaticano, 1966.

CS XVIII Opera omnia, vol. XVIII, Lectura II, dist. 1-6, ed. L. Modric et al., Typis Polyglottis Vaticanis, Ciudad del Vaticano, 1982.

CS XIX Opera omnia, vol. XIX, Lectura II, dist. 7-44, ed. L. Modric, Typis Polyglottis Vaticanis, Ciudad del Vaticano, 1993.

OPh III Opera philosophica, vol. III, Quaestiones super libros Metaphysicorum Aristotelis, libri I-V, ed. G.J. Etzkorn et al., The Franciscan Institute, Nueva York, 1997.

OPh IV Opera philosophica, vol. IV, Quaestiones super libros Metaphysicorum Aristotelis, libri VI-IX, ed. G.J. Etzkorn et al., The Franciscan Institute, Nueva York, 1997.

Wolter The Examined Report of the Paris Lecture. Reportatio I-A, Bychkov II vol. II, dist. 22-48, eds. A.B. Wolter y O.V. Bychkov, The Franciscan Institute, Nueva York, 2008.

Bibliografía secundaria

Cross, R., 2008, "Some Varieties of Semantic Externalism in Duns Scotus's Cognitive Psychology", Vivarium, vol. 46, no. 3, pp. 275-301.

Diánoia, vol. LXIII, no. 80 (mayo de 2018). 
Frank, W., 1992, "Duns Scotus on Autonomous Freedom and Divine Co-Causality", Medieval Philosophy and Theology, vol. 2, pp. 142-164.

González Ayesta, C., 2007, Naturaleza y voluntad, Eunsa, Pamplona.

Guzmán Manzano, I., 1990, "El principio de causalidad parcial en Escoto", Antonianum, vol. 62, nos. 2-3, pp. 290-311.

King, P., 2003, "Scotus on Metaphysics", en Williams 2003, pp. 15-68.

Ledsham, C., 2010, "Love, Power and Consistency: Scotus' Doctrines of God's Power, Contingent Creation, Induction and Natural Law", Sophia, vol. 49, no. 4, pp. 557-575.

Marrone, S., 2009, "Scotus at Paris on the Criteria for Scientific Knowledge", en S. Brown, T. Dewender y T. Kobusch (comps.), Philosophical Debates at Paris in the Early Fourteenth Century, Brill, Leiden, pp. 383-400.

Massie, P., 2011, Contingency, Time, and Possibility: An Essay on Aristotle and Duns Scotus, Lexington, Lanham.

Maurer, A., 1950, "Ens Diminutum: A Note on Its Origin and Meaning", Medieval Studies, vol. 12, pp. 216-222.

Pérez-Estévez, A., 2004, "Libertad en Duns Escoto", Revista Española de Filosofía Medieval, vol. 11, pp. 97-117.

Pini, G., 2009, "Scotus on the Possibility of a Better World", Acta Philosophica, vol. 18, no. 2, pp. 283-308.

Ross, J.F. y T. Bates, 2003, "Duns Scotus on Natural Theology", en Williams 2003, pp. 193-237.

Solaguren, C., 1968, "Contingencia y creación en la filosofía de Duns Escoto", Studia Scholastico-Scotistica, vol. 2, pp. 297-348.

Todisco, O., 1984, "Il metodo della concordanza e l'unione attitudinale dei fenomeni naturali", Studia Scholastico-Scotistica, vol. 8, pp. 301-320.

Vos, A., 2006, The Philosophy of John Duns Scotus, Edinburgh University Press, Edimburgo.

Vos, A. et al., 1994, "Lectura I 39. Commentary", en J. Duns Escoto, Contingency and Freedom. Lectura I 39, ed. A. Vos et al., Kluwer Academic Publisher, Dordrecht/Boston/Londres, pp. 43-189.

Williams, T. (comp.), 2003, Cambridge Companion to Duns Scotus, Cambridge University Press, Nueva York.

Recibido el 18 de agosto de 2016; revisado el 7 de abril de 2017; aceptado el 23 de octubre de 2017. 